\title{
IMPACT PARAMETER STUDY OF HIGH ENERGY ELASTIC SCATTERING*
}

\author{
Frank S. HENYEY, R. Hong TUAN ${ }^{\ddagger}$ and G.L. KANE \\ Physics Department, University of Michigan, Ann Arbor, Michigan 48104
}

Received 30 July 1973

(Revised 19 November 1973)

\begin{abstract}
The imaginary part of the proton-proton elastic scattering amplitude, as measured at the ISR, is examined in impact parameter. The transformed amplitude has two important properties. First, it is very accurately Gaussian in the impact parameter from the center to two fm with very little flattening near the center. Flattening would be expected from eikonalization. Secondly, there is a tail beyong two $\mathrm{fm}$ with a much flatter slope. This tail in impact parameter is equivalent to the break of $\mathrm{d} \sigma / \mathrm{d} t$ at $t \approx-0.15 \mathrm{GeV}$.

We discuss the physical origin of the tail. It cannot reasonably be diffraction dissociation, since diffraction should be large where absorption is large. We suggest that the tail is due to dissociation which must be distinguished from its diffractive part, and make a physical model which gives the tail and describes $\mathrm{d} \sigma / \mathrm{d} t$ very well. This model predicts the Deck model part of the diffraction inelastic cross section.

We discuss the interpretation of elastic scattering in terms of $s$-channel unitarity rather than a $t$-channel exchange or the structure of a single hadron.
\end{abstract}

\section{Introduction}

Most of the high energy elastic scattering amplitude is widely believed to be diffractive. That means different things to different people. For example, in the Regge frame-work, diffraction is defined as the exchange of a "Pomeron", To proceed from that viewpoint one transforms the energy dependence of the amplitude to $t$-channel angular momentum, and looks for singularities near $J=1$.

A more traditional viewpoint, which we shall adopt in this paper, is that diffraction is a consequence of s-channel unitarity. The structure of elastic scattering is supposed to be a reflection of all reactions which can occur between the initial particles. These viewpoints are not necessarily exclusive; it is likely to be profitable to study elastic scattering from both.

This latter viewpoint is more concerned with the momentum transfer dependence of the amplitude than its energy dependence. One way to proceed is to transform

\footnotetext{
* Research supported in part by the U.S. Atomic Energy Commission.

Fn leave from the Laboratoire de Physique Theorique et Hautes Energies, Orsay, France.
} 
to direct channel angular momentum, in which unitarity is diagonal. To simplify the calculations, and to exhibit the approximate energy independence of elastic scattering, one uses instead impact parameter which is essentially the same as angular momentum (i.e., $l=k b$ where $k$ is the c.m. momentum and $b$ is the impact parameter).

In this paper we do several related but different things. First we present the experimentally determined amplitude for high energy pp scattering as a function of impact parameter. The elements of structure in the amplitude measured as a function of $t$, namely the break at $t \approx-0.17$ and the dip at $t \approx-1.3$ give rise to structure of the amplitude as a function of impact parameter. We shall see that the break gives rise to a very long range part of the amplitude. We then describe the features of this structure which are relevant to any attempt at a physical interpretation in terms of $s$-channel concepts. We suggest such an interpretation and discuss difficulties with other interpretations. Our interpretation of the long range part is that it is caused by the possibility of dissociation of the incident and/or target particle into a larger system. At small impact parameter the entire amplitude has less structure than either the overlap function or the eikonal; we suggest that the small account of short range structure is absorption of the dissociative part. Finally we construct a simple model to exhibit our interpretation. This model although extremely crude, reproduces the structure in the experimental amplitude. Our model shares one feature with other attempts to explain the small $t$ break, namely that it is associated with a singularity in $t$. The origin of our singularity is physically different from other models, although not necessarily inconsistent with them.

Each part of the paper is based on the preceeding part. Thus the transform to impact parameter is relevant even if our interpretation is incorrect; the interprctation and speculations may be right even though our model cannot be an accurate approximation to the true situation.

\section{Impact parameter transformation}

We define the transformed elastic amplitude (helicity non-flip) as

$$
\tilde{M}(b)=\frac{1}{2} \int_{-\infty}^{0} M(t) J_{0}(b \sqrt{-t}) \mathrm{d} t / 2 q W,
$$

where $M(t)=M(s, t)$ is the usual amplitude and $q$ and $W$ are the center of mass momentum and total energy, respectively.

The differential cross section is

$$
\mathrm{d} \sigma / \mathrm{d} t=|M(t) / 2 q W|^{2} / 16 \pi,
$$


and the elastic cross section is

$$
\sigma_{\mathrm{el}}=\int_{-\infty}^{0} \mathrm{~d} t \mathrm{~d} \sigma / \mathrm{d} t=\int_{0}^{\infty} b \mathrm{~d} b|\tilde{M}(b)|^{2} / 8 \pi .
$$

The total cross section is

$$
\sigma_{\mathrm{T}}=\operatorname{Im} M(0) / 2 q W=\int_{0}^{\infty} b \mathrm{~d} b \operatorname{Im} \tilde{M}(b) .
$$

Unitarity is given at high energies by

$$
\operatorname{Im} \tilde{M}(b)=|\tilde{M}(b)|^{2} / 8 \pi+\tilde{O}(b) \text {, }
$$

where $\tilde{O}(b)$ is the overlap function [4] due to non-elastic $s$-channel intermediate states. The unitarity bounds are

$$
0 \leqslant \operatorname{Im} \tilde{M}(b) \leqslant 8 \pi, \quad 0 \leqslant \tilde{O}(b) \leqslant 2 \pi .
$$

For a given $\tilde{O}(b)$ and $\operatorname{Re} \tilde{M}(b)$, the quadratic unitarity equation (5) has two solutions for $\operatorname{Im} \tilde{M}(b)$. The smaller solution obeys

$$
0 \leqslant \operatorname{Im} \tilde{M}(b) \leqslant 4 \pi \text {. }
$$

We shall call this solution diffractive, as the larger solution corresponds to a $90^{\circ}$ phase shift as well as the absorptively generated scattering. There is no known reason that $90^{\circ}$ phase shifts should occur any more regularly than, say, $45^{\circ}$ phase shifts. In an optical picture the phase shift is given by the product of the index of refraction (real part) and the path length, and is independent of impact parameter only if the index of refraction is zero. Thus, a dominantly imaginary amplitude is very good evidence for a phase shift near zero and the smaller bound (8).

By combining eqs. (3-5) we find

$$
\sigma_{\text {inel }}=\sigma_{\mathrm{T}}-\sigma_{\mathrm{el}}=\int_{0}^{\infty} \tilde{O}(b) b \mathrm{~d} b=\int_{0}^{\infty} \tilde{O}(b) b \mathrm{~d} b=\left.\int_{0}^{\infty} \tilde{O}(b) b \mathrm{~d} b J_{0}(b \sqrt{t})\right|_{t=0} .
$$

The overlap function is the sum of contributions from each process with the given initial state. The cross section for each process is its contribution integrated over $b$.

We want to study mainly the dominant imaginary diffractive amplitude. To isolate it we work at the highest available energy, with the CERN ISR data [5-7] for pp scattering at $s=2800 \mathrm{GeV}^{2}$. It is possible that the cross section includes contributions also from the real part of the amplitude and from helicity flip amplitudes. The real part is probably as large as $10 \%$ of the imaginary part at some $t$ values near the forward direction, and for $-t$ near or beyond $1 \mathrm{GeV}^{2}$ the real part is presumably comparable to the imaginary part, probably filling in a zero in the imaginary part to make the dip at $1.4 \mathrm{GeV}^{2}$. We have tried several hypotheses for the real part and our 
results are quite insensitive to any reasonable choice. Basically that is because $\bar{M}(b)$ arises from an integral over $t$ which is dominated by the small $t$ cross section where the real part is of order a $1 \%$ (incoherent) effect. To be as realistic as possible we have used a reasonable model for the real part, but our results would not noticeably change if we chose zero real part. Similar statements apply to helicity flip contributions; since they are presumably present at the $1 \%$ level at $1500 \mathrm{GeV} / c$, they will have no qualitative effect on our results or our arguments. We have put them equal to zero. The only choice that can sifnificantly affect our results concerns the behavior of the imaginary part of the amplitude near the dip; does it have a single or a double zero? To our knowledge there is at present no compelling argument for either choice, but we are inclined to believe the amplitude has a single zero, arising from a cancellation between two physical mechanisms. The model we exhibit below will have this property and we will discuss it there in some detail. Miettinen and de Groot have considered the double zero case in a recent preprint [2].

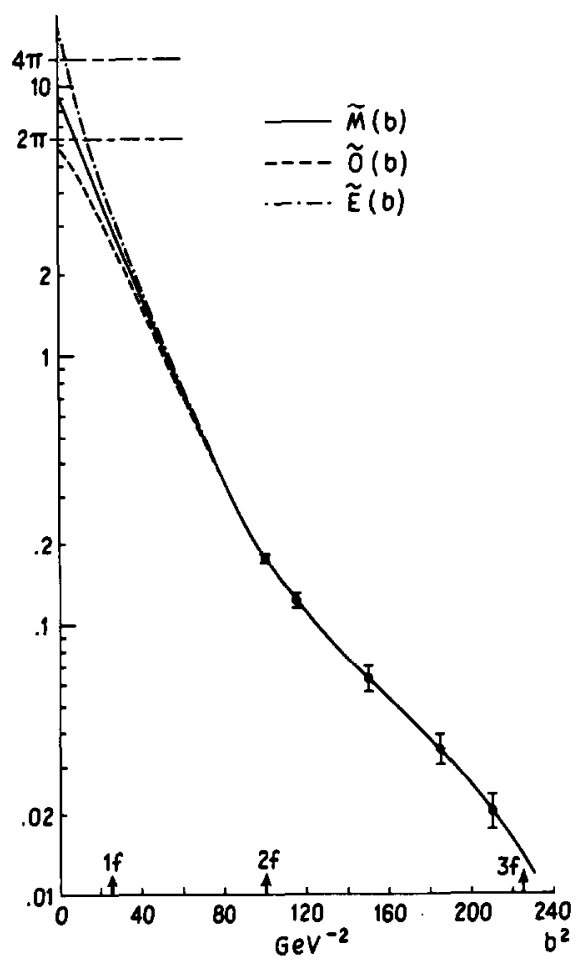

Fig. 1. The amplitude, overlap function, and eikonal extracted from experimental data as a function of impact parameter squared. (A straight line on this plot corresponds to an exponential in $t$.) The amplitude has a long tail between two and three fm, and continues to $b=0$ without a noticeable change in slope. These features are related to the break at small $t$ and the position of the dip at large $t$, respectively. 
Finally then, we calculate $\operatorname{Im} \tilde{M}(b)$ from

$$
\operatorname{Im} \tilde{M}(b)=\frac{1}{2} \int_{-\infty}^{0} \mathrm{~d} t J_{0}(b \sqrt{-t})\left(16 \pi\left(\mathrm{d} \sigma / \mathrm{d} t-[\mathrm{d} \sigma / \mathrm{d} t]_{R}\right)^{\frac{1}{2}},\right.
$$

where $(\mathrm{d} \sigma / \mathrm{d} t)_{R}$ is the real part contribution taken from ref. [8] and shown in fig. 7.

The result for $\operatorname{Im} \tilde{M}(b)$ is shown in fig. 1 , plotted against $b^{2}$. From eq. (5) we can calculate the overlap function, also shown in fig. 1 .

\section{Observations}

There are two striking features of $\operatorname{Im} \tilde{M}(b)$. First is the large $b$ tail. At about two $\mathrm{fm}\left(b^{2}=100\right)$, the curve levels off rather dramatically and maintains the shallower slope to about three $\mathrm{fm}\left(b^{2}=225\right)$; beyond that point the existing data without published numerical values is not good enough to see what is happening. The downward rounding around $b^{2}=180$ might be spurious; if not it is very interesting. The slope change suggests that the amplitude be considered as a sum of a short range piece and a long range piece $[8,9]$. The slope change occurs rather slowly near two $\mathrm{fm}$ so it is reasonable to extrapolate the tail in to smaller $b$ to give the long range piece.

The tail in $b$ is closely related to the sharp break observed in $\mathrm{d} \sigma / \mathrm{d} t$ at $-t \approx$ $\approx 0.15 \mathrm{GeV}^{2}$. We have transformed a number of different functions which closely approximated the amplitude apart from the small $t$ break and the tail was always absent in their transform. Models which try to reproduce the break without a tail (e.g., ref. [10]) do so at the expense of badly disagreeing with data for $t \geq 0.5$.

The second striking feature of the amplitude in impact parameter is that it is very nearly Gaussian near $b=0$. No bending is visible near $b=0$ in fig. 1 .

It has been widly hypothesized that elastic scattering would show structure due to eikonalization $[11,12]$; that is, at small $b$ the amplitude would be reduced because of its proximity to its limit of $4 \pi$. The eikonalization is given by

$$
\bar{M}(b)=4 \pi i(1-\exp (-\bar{E}(b) / 4 \pi i) \text {, }
$$

where $\tilde{E}(b)$ is the eikonal. ( $\tilde{E}(b)$ obeys the unitarity bound $\operatorname{Im} \tilde{E}(b) \geqslant 0$, and the overlap function is related to the eikonal by

$$
\tilde{O}(b)=2 \pi[1-\exp (-\operatorname{Im} \tilde{E}(b) / 2 \pi)] \text {. }
$$

The eikonal is $8 \pi$ times the phase shift.)

In looking at fig. 1 , one sees no evidence for any reduction near $b=0$ in $\bar{M}(b)$. If the eikonal were extrapolated from larger $b$ where it is small and approximately equal to the amplitude, assuming it were approximately Gaussian the amplitude calculated from eq. 11 would have an intercept of about 7 , compared to the true intercept of 9.25. An intercept of 7 would require a considerable flattening of the amplitude as it approached $b=0$, and would be quite visible in fig. 1 . 
By inverting eq. 11 we can find the eikonal which gives the observed amplitude,

$$
\tilde{E}(b)=4 \pi i \ln (1-\tilde{M}(b) / 4 \pi i) \text {. }
$$

$\tilde{E}(b)$ calculated in this way is also shown in fig. 1 . As can be seen, $\tilde{E}(b)$ must have considerable upward curvature near $b=0$. If onc believes that it is the eikonal which should be given by a model rather than the amplitude directly, then it must be considered an accident that the amplitude is so nearly Gaussian. It will be interesting to discover reasons for such rapidly varying eikonals. In any case, it is clear that eikonalization cannot be justified on grounds of simplicity.

On the other hand, the overlap function (fig. 1) does flatten out near $b=0$. If one believes that a model should describe the overlap function, then the central region must somehow be suppressed, e.g., by absorption. If the overlap function from larger $b$ were extrapolated as a Gaussian, its intercept would exceed its unitarity bound of $2 \pi$. In this case it need not be considered accidental that the amplitude is nearly Gaussian; the amplitude is the sum of multiparticle production and $|\tilde{M}|^{2} / 8 \pi$. The former could be simple and Gaussian but have its central region absorbed out. The latter is as central as absorption, so adding it back could reasonably produce the simple shape.

It is useful for studying the amplitude to note that it can be very well approximated by the sum of Gaussians

$$
\operatorname{Im} \tilde{M}(b)=8.94 \mathrm{e}^{-b^{2} / 21.3}+0.5 \mathrm{e}^{-b^{2} / 62.4}-0.239 \mathrm{e}^{-b^{2} / 0.92} .
$$

The first Gaussian dominates out to about two fm, beyond which the second takes over. The third is only present at small $b$, having to do with the dip at larger $t$, and can be ignored for the moment. This form exhibits the features we have described so far. The long range tail comes from the second term. The first has a mean radius of $0.8 \mathrm{fm}$ and the second a mean radius of $1.4 \mathrm{fm}$. The first term is a central contribution, while the second term provides a peripheral, edge contribution, as in refs. $[8,9]$. Near $b=0$ this sum of exponentials closely approximates the amplitude and is not to be corrected by eikonalization; the eikonal which gives the correct amplitude cannot be described very simply.

In momentum transfer eq. (13) becomes

$$
\operatorname{Im} M(t) / 2 q W=95.33 \mathrm{e}^{5.33 t}+15.6 \mathrm{e}^{15.6 t}-0.11 \mathrm{e}^{0.23 t} .
$$

The first exponential is larger than the second everywhere. The second is only important for very small $t,-t \lesssim 0.2$, and is responsible for the break. The comparison between eqs. (13) and (14) clearly shows the connection between the small $t$ break and the long $b$ tail.

Assuming the first exponential represents the contribution to diffraction of one type of multiparticle process, and the second to that of another type of multiparticle process, one sees that the second has a significantly smaller cross section than the first. From the $t=0$ intercepts one finds about $6 \mathrm{mb}$ for the second.

In fig. 2 we plot the slope of $M(b)$ extracted from data as a function of $b^{2}$; the 


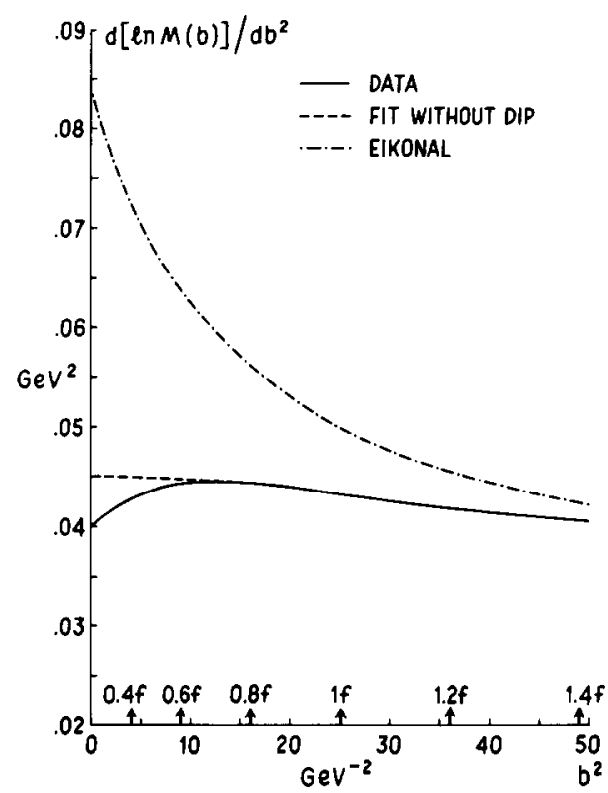

Fig. 2. The slope $\mathrm{d} \ln \widetilde{M}(b) / \mathrm{d} b^{2}$ as a function of $b$. There is a $10 \%$ decrease in the slope related to the dip at $t=-1.4$. The slope of the eikonal, $\mathrm{d} \ln \widetilde{E}(b) / \mathrm{d} b^{2}$, varies considerably. In an eikonal model it is an accident that the amplitude has so nearly constant a slope.

slope is given by $\mathrm{d} \ln \tilde{M}(b) / \mathrm{d} b^{2}$. A Gaussian would give a constant value for this slope. As $b$ approaches zero one can see a slight decrease, about $10 \%$. For comparison we show the slopes of the eikonal needed to produce the amplitude, and the slope of the first two exponentials which give a good fit to the data for $-t<1 \mathrm{GeV}^{2}$.

It is necessary to associate the negative term with one of the two positive exponentials because all processes must contribute positively at all $b[13]$. Since its size at $b=0$ is comparable to, but smaller than the second exponential, it is natural to associate it with that piece. Such an association is made both in the model we exhibit below and in the work of refs. [8,9] which treat the longer range piece as absorbed but the shorter range piece as not absorbed.

It is very important to realize that the negative contribution is very small. The rms size of the proton is about $1 \mathrm{fm}$. A diffractive amplitude for scattering of an object that size typically has a diffraction dip in the region $0.6 \mathrm{GeV}^{2} \lesssim-t \lesssim$ $\lesssim 0.8 \mathrm{GeV}^{2}$. On the other hand, the pp scattering has its zero at $t=-1.4 \mathrm{GeV}^{2}$ where the amplitude is two orders of magnitude smaller than at 0.6 . The negative exponential would have to be about 25 times larger to move the dip in to $0.6 \mathrm{GeV}^{2}$. Thus in a sense the dip is nearer to $-t=$ infinity than to $-t=0.6$.

This is illustrated in fig. 3 where we show two eikonals which are similar, one giving an amplitude with a dip at $-t=1.4 \mathrm{GeV}^{2}$, the other no dip at all. There is no obvious way to choose between them except to know the data: 


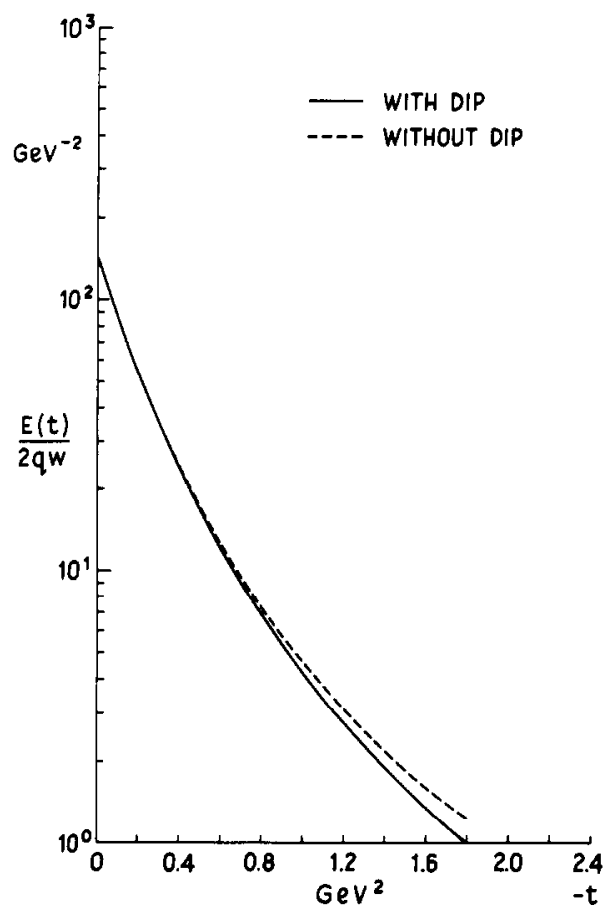

Fig. 3. Two possible eikonals. One gives a dip at $t=-1.4$, and the other gives no dip. They are so nearly alike that only the data allows one to choose the correct one. Thus, in an eikonal type model the position of the dip is essentially unpredictable.

All of this discussion of the negative contribution is, of course, dependent on our decision to choose the imaginary part to be negative beyond the dip. If it is positive, clearly a three exponential fit is inadequate.

\section{Interpretation}

So far we have been led by the data to a two component picture ${ }^{\dagger}$ of elastic scattering. There is a central component, large in size, with no apparent structure, and there is a peripheral component, small in size, extending well beyond the central component. The peripheral component appears to be absorbed. Our observations are largely phenomenological and model independent. Now we turn to speculations on the origins of these components, and interpretations.

$\dagger$ As is explained in the text, our two components (peripheral and other) do not correspond exactly to the usually defined two components (diffraction and other) of Wilson [14] and many recent works. 
What processes, via unitarity, contribute to the two pieces? In particular, what gives rise to the contribution at large $b$ ? Since unitarity is diagonal in $b$, it must be processes which are themselves spread out. The mechanism which suggests itself is that a hadron can virtually dissociate into a system of two or more particles, and the system occupies more area than the original proton [15]. One of the fragments then strikes the other proton (or a piece of it). The impact parameter statement that the dissociated system has a larger area than the original proton is equivalent to the momentum space statement that the fragment which interacts with another hadron has a propagator and a form factor. (Either one alone causes spreading.)

The absorption of the dissociation piece can be understood as the possibility that, for example, a proton dissociates into two fragments and both fragments interact with the other proton. This possibility has been taken into account in the central

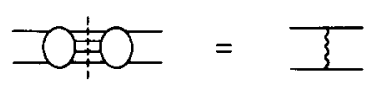

(a)

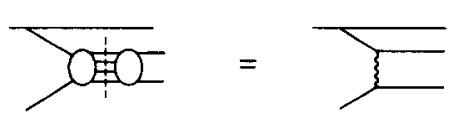

(b)

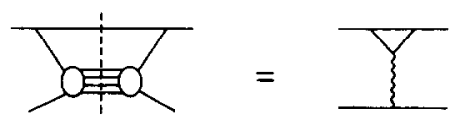

(c)

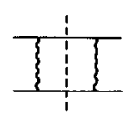

(d)

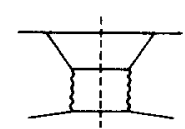

(e)

Fig. 4. s-channel unitarity mechanisms for elastic scattering and diffraction dissociation, concentrating on the peripheral component. (a) All scattering builds up elastic scattering. (b) All dissociative scattering builds up diffraction dissociation as given by the Deck model. (c) All dissociative scattering also contributes to elastic scattering. This is a part of that shown in (a). We identify the dissociative part with the peripheral component. (d) The contribution of elastic scattering is only part of the total shown in (a), (although the largest single piece). It has a shorter range than the total. (e) Analogous to (d), diffraction dissociation is a contribution to the dissociative component of (c), and has shorter range than all of (c). 
component with the possibility that the entire proton interacts with the other. This sort of effect is familiar from absorption calculations.

A very important distinction must be made between dissociation processes and diffraction dissociation, analogous to the distinction between all scattering and elastic scattering. The imaginary part of elastic scattering is built up by contributions from all scattering (fig. 4a). The contribution of elastic scattering itself is only one contribution out of many (fig. 4d), although it is the largest single contribution. In the same way, all dissociative scattering builds up diffraction dissociation (fig. 4b), diffraction dissociation being but one contribution. Dissociative scattering also contributes to elastic scattering (fig. 4c). Diffraction dissociation is just one part of it (fig. $4 \mathrm{e}$ ).

It would be unreasonable to assume that the peripheral component was caused by diffraction dissociation alone. Diffraction is the shadow of other scattering. Thus, if there is no other scattering, there cannot be diffractive scattering either. Between two and three fm (fig. 1) the peripheral part is much larger than the rest, and therefore is not diffractive ${ }^{\dagger}$.

\section{Model}

We can make a crude model incorporating the mechanism we propose. The model is not meant to be complete either theoretically or phenomenologically. Rather, it shows simply and clearly how the features we have described can give rise to the structure seen in elastic scattering.

Assume the central contribution to $M(s, t)$ is given by a known function $H(s, t)$; for numerical work we will choose $H(s, t)=i s A_{1} \mathrm{e}^{B_{1} t}$. All the amplitudes are treated as purely imaginary elastic ones. Similarly, the dissociation component of elastic scattering, shown in fig. 5 , is given by an integration

$$
\int \frac{\mathrm{d}^{3} k}{E_{1}} G\left(t_{1}\right) G\left(t_{2}\right) M\left(s_{2}, t\right),
$$

where $G(t)$ describes the dissociation vertex and propagator of the virtual fragment which interacts with the target. We estimate the integral using an exponential in $t$ for $G(t)$. Essentially all the integral comes from $s_{2}$ being proportional to $s$, and the integrand is moderately strongly peaked at $t_{1}+t_{2}$ a fixed fraction of $t$. The integral can then be approximated by

$$
M(s, t) A_{2} \mathrm{e}^{B_{2} t} .
$$

Thus the amplitude satisfies

$$
M(s, t)=H(s, t)+M(s, t) A_{2} \mathrm{e}^{B_{2} t},
$$

$\dagger$ The opposite opinion has been expressed by Barshay [16]. 


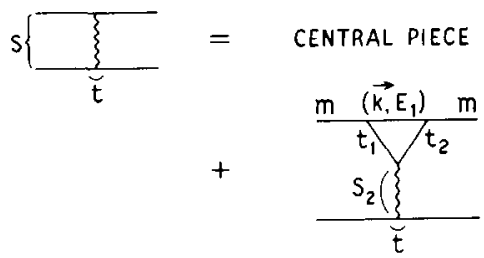

Fig. 5. Diagrammatic explanation of our mechanism for elastic scattering. The amplitude is the sum of a relatively structureless central component and a dissociative component. We have not illustrated the absorption of the dissociative component. The kinematics of dissociation is shown.

or

$$
M(s, t)=H(s, t) /\left(1-A_{2} \mathrm{e}^{B_{2} t}\right)=H(s, t)+H(s, t) A_{2} \mathrm{e}^{B_{2} t} /\left(1-A_{2} \mathrm{e}^{B_{2} t}\right) .
$$

Finally, according to our physics arguments above, we must effectively absorb the dissociation component, which can be identified with the second term in eq. 17; the central part is given by $H(s, t)$. This can be done by first expanding

$$
M(s, t)-H(s, t)=I A_{2} \mathrm{e}^{B_{2} t}\left(1+A_{2} \mathrm{e}^{B_{2} t}+\ldots\right),
$$

then transforming the entire equation to impact parameter, then absorbing the central partial waves by multiplying each term by

$$
\tilde{S}_{\text {eff }}(b)=1-\mathrm{e}^{-b^{2} / 4 B_{1}} \text {, }
$$

where the right hand side gives the simple form used for numerical work, transforming the whole series back to the momentum transfer variable and resumming. The amplitude for our model can be written in the form

$$
M(s, t)=H(s, t)+\left(H(s, t) A_{2} \mathrm{e}^{B_{2} t} /\left(1-A_{2} \mathrm{e}^{B_{2} t}\right)\right) * S_{\text {eff }}
$$

where * means an impact parameter convolution as explained above.

The parameters $A_{1}, A_{2}, B_{1}, B_{2}$ are adjusted to best fit the data, giving the results shown in figs. 6,7 . The decomposition into central plus dissociative is shown in figs. 6, 7. The parameter values are $A_{1}=80.7, B_{1}=4.32, A_{2}=0.35, B_{2}=3.09$.

The entire cross section can be broken down into the individual pieces shown in fig. 4 , i.e., the individual amplitudes at $t=0$. We find that the entire cross section of $43.1 \mathrm{mb}$ is made of $31.4 \mathrm{mb}$ of central component and $11.7 \mathrm{mb}$ of dissociative component. The central component includes $7.6 \mathrm{mb}$ of elastic scattering (eq. (3)). The dissociative component includes $1.7 \mathrm{mb}$ of diffraction dissociation. This calculation is the diagram of fig. 4e. Fig. $4 \mathrm{~d}$ is calculated by an impact parameter convolution, the result is multiplied by $A_{2} \mathrm{e}^{B_{2} t}$ to approximate the loop integral and then this is absorbed and evaluated at $t=0$.

The value $1.7 \mathrm{mb}$ for diffraction dissociation is smaller than that usually estimated [18] at NAL and ISR energies. This may be caused by the severe approximations in our model. Another possible explanation is more physical. Our diagrams (fig. 4b) for 




Fig. 6. Model fit to the amplitude, shown in impact parameter. The decomposition into the two terms of fig. 5 is shown. The dissociative component is long range and absorbed.

diffraction dissociation correspond to the Deck model, and have the feature that the intermediate state to final state scattering is disconnected. However, non-Deck diffraction inelastic processes can occur in which the intermediate to final state scattering is connected. In these processes the initial state becomes a non-dissociated intermediate state, which then becomes a final state slightly different from the initial state. These processes are not properly dissociative; they should be referred to as direct diffraction inelastic rather than diffraction dissociation. In our model, such processes are to be included in the central component. Therefore our $1.7 \mathrm{mb}$ is only an estimate of the Deck type diffraction dissociation which is to be added to the direct diffraction inelastic (if any) to give the total experimentally estimated diffraction inelastic. In other words, perhaps the experimental diffraction inelastic includes more than our theoretically defined diffraction dissociation. In this connection it is interesting to note that the experimental data usually called diffraction dissociation often show a break in slope [17] at $-t \sim 0.2 \mathrm{GeV}^{2}$ with about half the area under a (peripheral) piece with steep slope (slope greater than elastic) and about half under a shallow piece (slope about half elastic). It may be that the correct interpretation will be to identify only one part of the usual curve with the (Deck type) diffraction 




Fig. 7. Model fit to the amplitude, shown in momentum transfer. The decomposition into the two terms of fig. 5 is shown.

dissociation part of our model and the other part with the direct diffraction inelastic.

By expanding the solution in powers of $A_{2}$ one can separate the contributions of fragmentation into $n$ hadrons, which is important for understanding the tail as large $b$. In the fit shown in figs. 6 and 7 , the two fragments dissociation is less than half of the total dissociation beyond two $\mathrm{fm}$. Thus the multifragment processes are phenomenologically important. The dissociation curve in fig. 6 is approximately given by including the dissociation with four or less fragments suggesting a reasonable (low) multiplicity for the dissociation part of the cross section.

Since we include all numbers of fragments, the infinite series generates a singularity in $t$. In our model this singularity is a pole (see eq. 17 or 19) at $A_{2} \mathrm{e}^{B_{2} t}=1$. With the parameters of our fit, this pole is at $t=+0.26 \mathrm{GeV}^{2}$. This pole should not, of course, really exist in the amplitude and will be replaced by a soft singularity in a model with less approximations.

Although the pole is not reasonable from a fundamental theoretical viewpoint, it is extremely reasonable as an approximation. There are singularities in $t$ which 
really do exist in the amplitude, the two pion threshold, the two rho threshold, the $\mathrm{N} \overline{\mathrm{N}}$ threshold etc. Our pole should be interpreted as an effective singularity, approximating all the singularities which actually exist. In a sense, the $t$-singularities are responsible for the structure at small $t$.

A singularity in $t$ is responsible for the structure at small $t$ in other approaches. For example, Anselm and Gribov [3] suggest that the $\pi \pi$ threshold is responsible for the break. However it has not been shown that this effect is sufficient to give the magnitude and shape of the small $t$ cross section. As another example, the model of Cheng and $\mathrm{Wu}[12]$ contains a singularity at a position which they take to be an adjustable parameter. In a model such as our which admits being crude, it is reasonable that the position of the effective singularity be an adjustable parameter or an output from fitting data. In a model such as that of Cheng and Wu which makes a claim to be a much better approximation it would seem to be appropriate that the two pion threshold be the singularity nearest $t=0$. (Cheng and Wu discuss the limit of extremely large impact parameter which they claim to be relevant. It is clear that the $2 \pi$ threshold dominates as $b \rightarrow \infty$.)

The only implication of our model that might give trouble (which we are aware of) is the connection with diffractive production on nuclei [19]. The question is a complicated one, which will require careful calculations. Basically we have given dissociated hadrons a larger size than undissociated ones. In the present crude model this spreading is too large to allow the shadowing of the fragments to reduce the cross section to that observed. In a version of the model which gives a large diffraction dissociation, the spreading will be smaller, and the cross section in nuclei will be smaller.

We would like to make one observation concerning energy dependence. Our analysis has been done in impact parameter, and is essentially unconnected with $s$ dependence. However, our interpretation in terms of dissociation states suggests a way to look at the situation [15]. Note that at $1500 \mathrm{GeV} / c$ in fig. 7 the full curve is the sum involving 1,2, and 3 dissociations. At a lower energy presumably the contribution with three dissociations is less probable because it requires considerable phase space. Then the tail in $b$ could not extend out so far and the small $t$ break would not be so pronounced. In addition, $\sigma_{\mathrm{T}}$ could increase with increasing energy as more dissociative states are produced. Unfortunately, we cannot say whether the energy dependence effect we are noting here is numerically large enough to matter because we have no dynamical energy dependence in the model.

\section{Summary}

We would like the reader to remember the following points. The experimental data, looked at in impact parameter $b$, shows two properties which must be present in any interpretation. These are (i) the surprisingly long tail in $b$, which is directly related to the small $t$ slope increase, and (ii) the lack of any large deviation from a 
pure Gaussian shape in $b$ at small $b$, which is directly related to the $\operatorname{dip}$ in $\mathrm{d} \sigma / \mathrm{d} t$ occurring at a large $t$ where the cross section is very small (rather than where a diffraction minimum would occur). The dip is almost at $-t \rightarrow \infty$, as indicated by the fact that eikonalizing with form factor structure gives a dip while eikonalizing with a slightly different eikonal gives no dip.

We have tried to interpret these phenomena from the viewpoint of $s$-channel unitarity and physical particle states. The model we have exhibited describes the large tail in $b$ as due to dissociation of hadrons; the dissociated states are spread out. An important point is that the dominant contribution to the dissociation cross section is not diffraction dissociation; diffraction dissociation is the shadow of the other processes that can occur and will always be less than half (much less at large $b$ ) of the cross section at a given $b$ just as diffractive elastic scattering will be less than half of $\sigma_{\mathrm{T}}$, unless direct diffractive inelastic processes occur. The dip at $-t \simeq 1.4$ $\mathrm{GeV}^{2}$ arises in our interpretation because the dissociation contribution should come from the edge region of the hadron interactions; this can be thought of as a peripheral interaction when the central region of the dissociation is absorbed away. Then the destructive contribution which causes the dip is smaller because it is the absorption of the dissociation part $\left(\sigma_{\text {diss }} \sim \frac{1}{4} \sigma_{\mathrm{T}}\right)$ rather than of the entire amplitude, so the dip is moved out in $-t$ compared to what would be expected from a diffraction zero. Our interpretation leads to some incomplete speculation on energy dependence because the dissociation mechanism is inhibited at any finite energy.

We would like to thank Marc Ross for comments and for careful reading of the manuscript.

\section{References}

[1] U. Amaldi, CERN report 73-5 (April, 1973).

[2] E.H. de Groot and M.I. Miettinen, Rutherford Lab. preprint RL-73-003.

[3] A.A. Anselm and V.N. Gribov, Phys. Letters 40B (1972) 487.

[4] L. Van Hove, Rev. Mod. Phys. 36 (1964) 655.

[5] G. Barbiellini et al., Phys. Letters 39B (1972) 663.

[6] U. Amaldi et al., (CERN-Rome Collaboration) Phys. Letters B44 (1973) 112 ; S.R. Amendolia et al., (Pisa-Stonybrook Collaboration), Phys. Letters B44 (1973) 119.

[7] ACHGT Collaboration (unpublished data).

[8] G.L. Kane, Phys. Letters 40B (1972) 363;

M. Vaughn and G.L. Kane, to be published.

[9] V. Barger, R.J.N. Phillips and K. Geer, Nucl. Phys. B47 (1972) 29.

[10] P. Heckman and R. Henzi, Phys. Letters 41 B (1972) 189.

[11] T.T. Chou and C.N. Yang, Phys. Rev. 170 (1968) 1591;

L. Durand III and R. Lipes, Phys. Rev. Letters 20 (1968) 637;

B. Carreras and J.N.J. White, Nucl. Phys. B42 (1972) 95.

[12] H. Cheng and T.T. Wu, Phys. Rev. Letters 26 (1970) 1456.

[13] F.S. Henyey, Phys. Letters, to be published.

[14] K. Wilson, Cornell report CLNS-1 31 (unpublished, 1970). 
[15] L. Stodolsky, SLAC-PUB-864 (1971) (unpublished).

[16] S. Barshay, Nuovo Cimento Letters 3 (1972) 369, and ref. [2].

[17] J.C. Sens, Invited Paper at the Conf. on recent advances in particle physics, New York Academy of Sciences, April 1973;

F.T. Dao et al., NAL preprint 73/22-EXP.

[18] J. VanderVelde, Contribution to the Proc. of the 16th Int. Conf. on high energy physics, (NAL 1972), p. 260;

J.C. Sens, Contribution to the Proc. of the 16th Int. Conf. on high energy physics (NAL) 1972 , p. 260.

[19] W. Beusch, Acta Phys. Pol. B3 (1972) 679.

[20] G.F. Chew, Berkeley preprint LBL-1556 (January 1973); Phys. Letters 44B (1973) 169;

A. Capella and Min-Shih Chen, SLAC preprint 1252 (May 1973);

D. Amati, L. Caneschi and M. Ciafaloni, CERN preprint 1676 (May 1973). 